\title{
Determination of Protein Titration Curves Using Si Nanograting FETs*
}

\author{
Silu Zhang, Student Member, Pengyuan Zang, Student Member, Yuchen Liang, Student Member, and \\ Walter Hu, Senior Member, IEEE
}

\begin{abstract}
This study describes a methodology of measuring protein titration curve by Si Nanograting FETs (NGFETs). Two proteins were measured with each of the titration curves showing specific characters for identification. The measurements at high ionic concentration were performed to eliminate the effect of $\mathrm{SiO}_{2}$ surface. The intrinsic protein titration curves extracted are consistent with prior researches.
\end{abstract}

\section{INTRODUCTION}

The net charges of protein is an important feature that has been drawn great attention in biomolecular researches.[1-5] The titration curve, which is known as the protein surface net charge behavior as a function of $\mathrm{pH}$ values, has been theoretically computed based on the Henderson-Hasselbalch equation $[3,4]$. As the shape of the curve is determined by the acid dissociation constant $(\mathrm{pKa})$ of each amino-acid residue, the titration curve can be presented as a representative of certain type of protein. Therefore, determination of titration curve is of great value in terms of the potential in identifying proteins. Surface charge is typically measured by zeta potential $[5,6]$. R. Kun et al. studied the net charge of bovine serum albumin (BSA) by applying titration with polyelectrolytes in the Mütek Particle Charge Detector[7]. However, through these methods it can barely get enough data points to form an accurate titration curve.

Silicon-based field effect transistors (Si FETs) have been proved to be effective biosensors in detecting surface charges (or potential).[8] Among various types of FETs, $\mathrm{Si}$ Nanograting FETs (Si NGFETs) shows better stability.[9] P. Zang et al. have shown the outstanding performances of NGFETs as a pH sensor.[10]

A new methodology was applied in this study to determine the titration curve of proteins via NGFETs. It was demonstrated to be a convenient method providing more accurate outcomes. Firstly, by showing the respective titration curves of BSA and immunoglobulin $\mathrm{G}$ from mouse serum (mouse $\mathrm{IgG}$ ), the feasibility of distinguishing two types of proteins was proved. Secondly, the effect cause by $\mathrm{SiO}_{2}$ surface of the device was measured at high ionic strength and could, therefore, be eliminated to extract intrinsic protein characteristic. Finally, the outcome matches with the simulating results and also can be verified by the zeta potential data.[5]

*All authors are with the Electrical Engineering Department, University of Texas at Dallas, Richardson TX 75080, (Walter Hu, phone: 972-883-6329; e-mail: walter.hu@utdallas.edu).

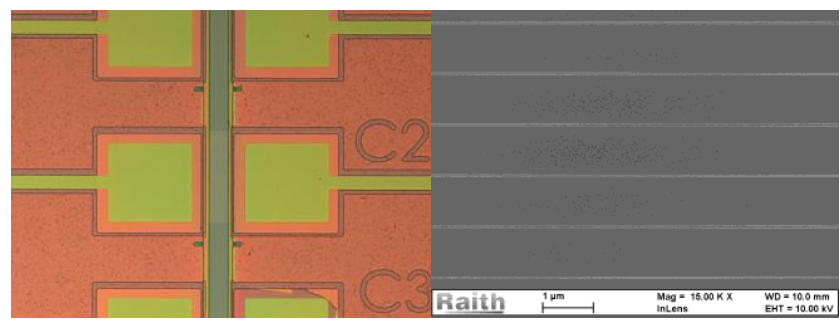

Figure 1. Schematics of NGFETs: a) Optical image; b) Scanning electron microscope (SEM) image of nanowire channels.

\section{MATERIALS AND METHODS}

\section{A. NGFETs Fabrication}

The schematic of the n-type NGFET used in this study is shown in Fig. 1. The nanowires with 3-nm silicon dioxide grown on the surfaces are designed as $20 \mu \mathrm{m}$ in length, $50 \mathrm{~nm}$ in width and $30 \mathrm{~nm}$ in height. This dimension of nanowires was chosen to achieve optimal device sensitivity.[11] The channel consists of 100 nanowires and was proved to have better device performance in stability.[9] The detailed fabrication process of NGFETs is described in previous work $[9,10]$.

\section{B. Surface Modification}

The surfaces of NGFETs have been treated by piranha $\left(\mathrm{H}_{2} \mathrm{O}_{2}: \mathrm{H}_{2} \mathrm{SO}_{4}=1: 3\right)$ for $30 \mathrm{~min}$ and dried with $\mathrm{N}_{2}$. To coat the surface with self-assembled monolayer (SAM) for protein immobilization, the device was immersed in $0.1 \%$ 11-(triethoxysilyl) undecanal (TESU, Gelest, Inc) in toluene for 5 hours and dried with $\mathrm{N}_{2} .50 \mu \mathrm{g} / \mathrm{mL}$ mouse IgG or BSA (both from Sigma-Aldrich) was dissolved in in $2 \mathrm{mM}$ PBS containing $1 \mathrm{mM} \mathrm{NaCNBH}$ and reacted with TESU modified surface for $3 \mathrm{hs}$, followed with rinsing by $2 \mathrm{mM}$ PBS. The device was stored in a sealed holder with its surface covered by $2 \mathrm{mM}$ PBS before test.

TABLE I. REPRESENTATIVES OF FunCTION GROUP CHARGE STATUS

\begin{tabular}{|c|c|c|c|}
\hline \multirow{2}{*}{ Function Group } & \multicolumn{3}{|c|}{ Charge Status } \\
\cline { 2 - 4 } & Positive & Neutral & Negative \\
\hline Hydroxyl & $-\mathrm{OH}_{2}^{+}$ & $-\mathrm{OH}$ & $-\mathrm{OH}^{-}$ \\
\hline Adehyde & $-\mathrm{CH}_{2} \mathrm{O}^{+}$ & $-\mathrm{CHO}$ & $-\mathrm{COO}^{-}$ \\
\hline Amino & $-\mathrm{NH}_{3}^{+}$ & $-\mathrm{NH}_{2}$ & \\
\hline Thiol & & $-\mathrm{SH}$ & $-\mathrm{S}^{-}$ \\
\hline
\end{tabular}




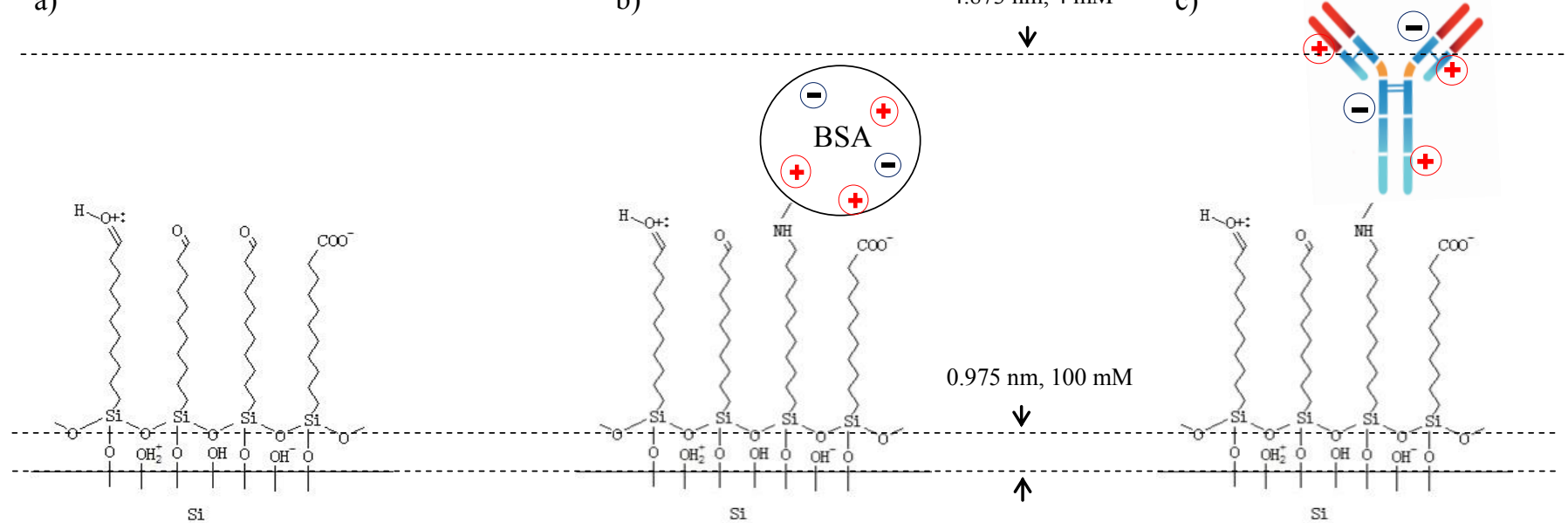

Figure 2. Schematics of different surface modification on NGFETs: a) TESU only; b) TESU coated with BSA; c) TESU coated with mouse IgG.

\section{Electrical Measurement}

PBS buffers with various $\mathrm{pH}$ values were selected by a selector valve and were delivered to the gate of the sensor through PDMS channel at a flow rate of $100 \mu \mathrm{L} / \mathrm{min}$. The fluidic pressure at 5 psi was supplied by a nitrogen gas cylinder, so as to eliminate the effect of $\mathrm{CO}_{2}$ dissolution to ensure a constant $\mathrm{pH}$ value for each buffer during test. The gate voltage was applied to solutions through an $\mathrm{Ag} / \mathrm{AgCl}$ electrode at a proper potential where the sensor worked in its sub-threshold region. The conductance of device at this time can respond to changes in surface potential. For n-type NGFETs as used in this study, increased surface (or gate) potential accumulates carriers in the channel and therefore cause increase in drain currant, while decreased surface potential depletes carriers and reduce drain current. The drain current was measured and recorded by Keithley 4200-SCS. Buffer of each $\mathrm{pH}$ value was injected to the sensor for $\sim 100 \mathrm{~s}$ to obtain a stable signal. Each titration curve was obtained by averaging two sweeps.

\section{RESULTS AND DISCUSSION}

Three different NGFETs surfaces shown in Fig. 2 were studied. The $\mathrm{SiO}_{2}$ surface was hydroxyl-terminated after the piranha treatment. In Fig. 2a, TESU molecules were assembled to the surface through forming $\mathrm{Si}-\mathrm{O}$ bonds and having $-\mathrm{CHO}$ terminals to anchor the protein of study. The amino groups of each protein reacted with $-\mathrm{CHOs}$ to form Schiff bases and then reduced by $\mathrm{NaCNBH}_{3}$, as depicted in Fig. $2 b$ and c. Part of the $-\mathrm{CHOs}$ which have not reacted with the proteins were possibly oxidized to $-\mathrm{COOH}$ by the $\mathrm{O}_{2}$ in the air. Therefore, the net charge of the surface was contributed by the combination of protonated or deprotonated $-\mathrm{OH},-\mathrm{CHO},-\mathrm{COOH}$ and residues of protein molecules. The charge status of main functional groups are presented in TABLE I. In low $\mathrm{pH}$ environment, protonation tended to dominate to have functional groups positively charged; whereas in high $\mathrm{pH}$ environment, deprotonation was the dominant factor for functional groups being negatively charged. At certain $\mathrm{pH}$ value, which is called isoelectric point (pI), the extent of protonation equals to deprotonation, results in the net charge at the surface equal to zero.
However, not all charges of the multi-component surface can be sensed by the device. Only those that are close enough to the device surface can be detected, others are screened. The detectable distance is set by Debye length, which is determined by ionic concentrations of solutions. So by measuring at a specific ionic concentration, the region above the surface within a given distance can be selectively detected. The detectable region given by Debye length and its corresponding ionic concentration is schematically marked in Fig. 2 (Dimensions are not scaled). The orientation of each protein is arbitrary instead of necessarily in the status shown in Fig. 2. Therefore, even though a protein molecule may be larger than $5 \mathrm{~nm}$ in height, it is still sensible as being partially lying in the detectable region.

\section{A. Titration Curve Measured at Low Ionic Concentration}

Titration curves for each of the three surfaces described in Fig. 2 were consistently measured at $4 \mathrm{mM}$ ion concentration and are shown in Fig. 3a. The current was converted to surface potential according to $\mathrm{Id}-\mathrm{Vg}$ curve of NGFET. Since theoretical Debye length at this concentration is $4.873 \mathrm{~nm}$ [11], charges of $\mathrm{SiO}_{2}$, TESU and proteins can all contribute to the signal detected by device. The plots in Fig. $3 a$ are the titration curves of the three surfaces, respectively. Despite the effect of $\mathrm{SiO}_{2}$ surface, these curves exhibited different shapes that were distinguishable from each other. The behavior at the ends of each titration curve reflects a screening effect that matches the simulation result in Yang's work. At low/high $\mathrm{pH}$ values, the hydroxyl groups of $\mathrm{SiO}_{2}$ surface are largely charged due to extensive protonation/deprotonation. The charges above the TESU molecular layer are, hence, screened by the highly

TABLE II. Key VALUES OBtAINED From Fig. 3

\begin{tabular}{|c|c|c|c|}
\hline \multirow{2}{*}{ Curve Legend } & \multicolumn{3}{|c|}{ Key Values } \\
\cline { 2 - 4 } & $\boldsymbol{p K \boldsymbol { a } , \boldsymbol { 1 }}$ & $\boldsymbol{p} \boldsymbol{K} \boldsymbol{a}, \boldsymbol{2}$ & $\boldsymbol{P I}$ \\
\hline TESU_100 mM & 4.0 & 10.0 & 7.0 \\
\hline TESU_4 mM & 5.0 & 9.2 & 7.1 \\
\hline TESU+BSA_4 mM & 3.4 & 9.2 & 6.3 \\
\hline
\end{tabular}




\begin{tabular}{|c|c|c|c|}
\hline \multirow{2}{*}{ Curve Legend } & \multicolumn{3}{|c|}{ Key Values } \\
\cline { 2 - 4 } & $\boldsymbol{p K a}, \mathbf{1}$ & $\boldsymbol{p K a}, \mathbf{2}$ & $\boldsymbol{P I}$ \\
\hline TESU+IgG_4 mM & 4.3 & 9.3 & 6.8 \\
\hline
\end{tabular}

a)

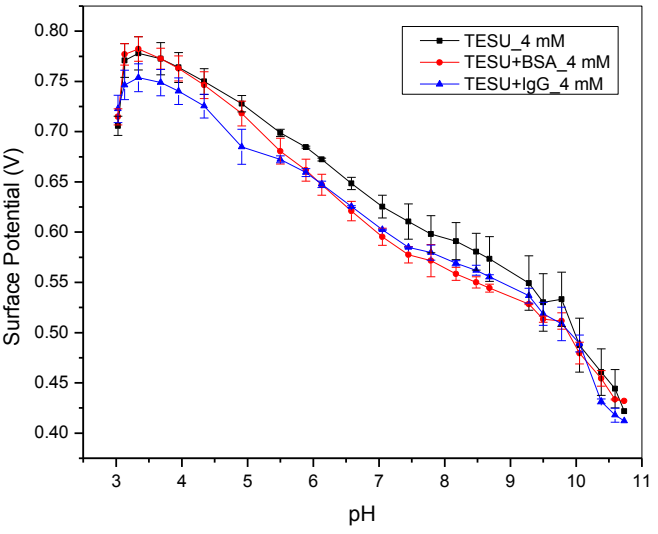

b)

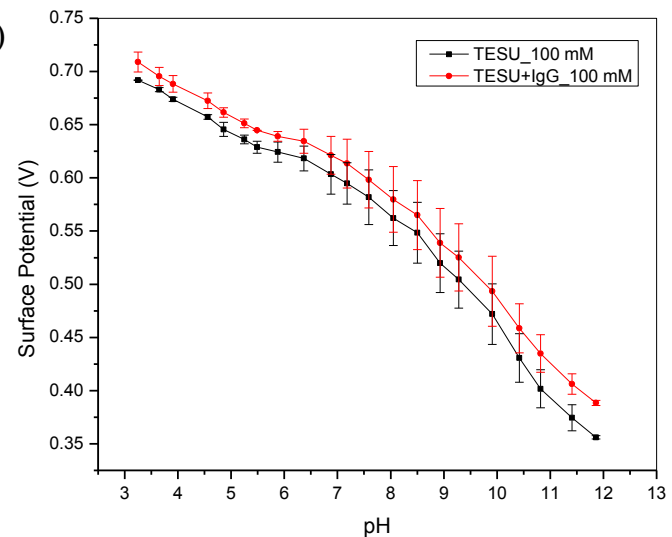

Figure 3. Titration curve measured at ionic concentration of a) $4 \mathrm{mM}$ and b) $100 \mathrm{mM}$.

charged $\mathrm{SiO}_{2}$ surface. In the range of low $\mathrm{pH}$ values $(<3.5)$, increasing amount of positive charges are screened with the declining $\mathrm{pH}$ values. As a result, the surface potential drops as the screening effect dominates. Similar behavior appears in the range of high $\mathrm{pH}$ values $(>10.5)$.

\section{B. Titration Curve Measured at High Ionic Concentration}

To further observe the intrinsic $\mathrm{pH}$ dependent behavior of BSA and mouse IgG, the impacts of $\mathrm{SiO}_{2}$ surface and uncovered TESU terminal groups were eliminated from the titration curves. To achieve this, the titration curve of TESU modified surface was measured at $100 \mathrm{mM}$ ionic concentration with the Debye length reported as 0.975 $\mathrm{nm}[11]$, so the potential change was only from the functional groups existing within the height of $1 \mathrm{~nm}$ above the surface. The result is shown as the black curve in Fig. 3b. No screening effect was observed at the two ends because charges at the higher position of the layer had been screened by high concentration of slat in the entire $\mathrm{pH}$ region. As expected, the curve reflects the intrinsic behavior of hydroxyl group at the $\mathrm{SiO}_{2}$ surface and is therefore different from the black curve in Fig. 3a. The result was verified by taking same measurement on mouse IgG modified surface. The outcome is shown as the red curve in Fig. 3b. The curve has identical shape as that of TESU modified surface, due to the fact that the higher portion of the surface molecule layer failed to give a signal.

a)

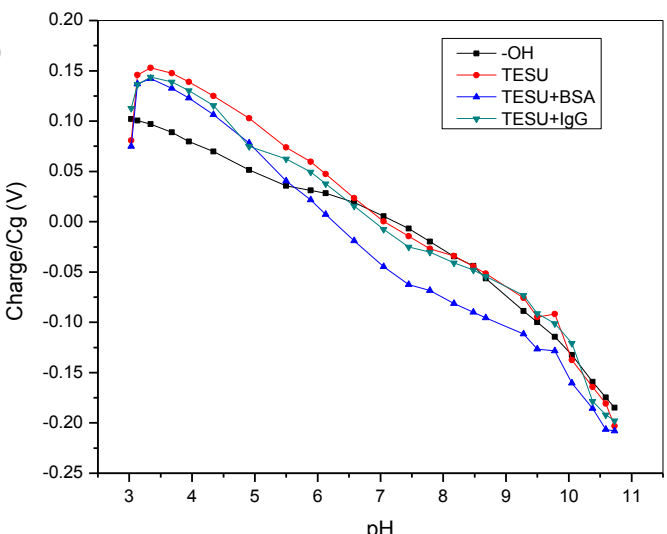

b)

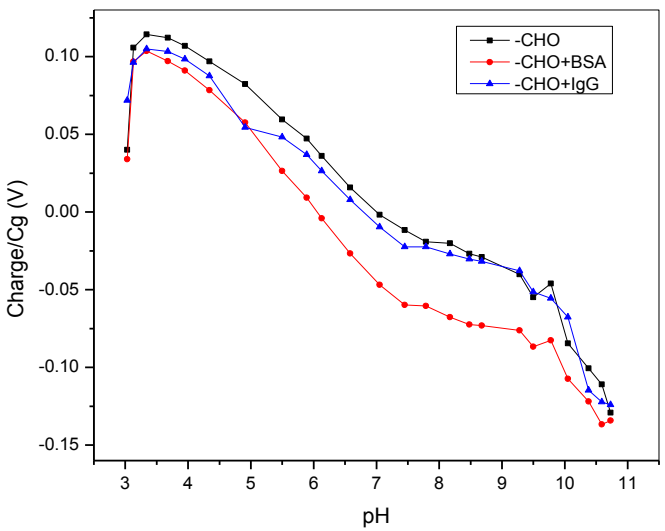

c)

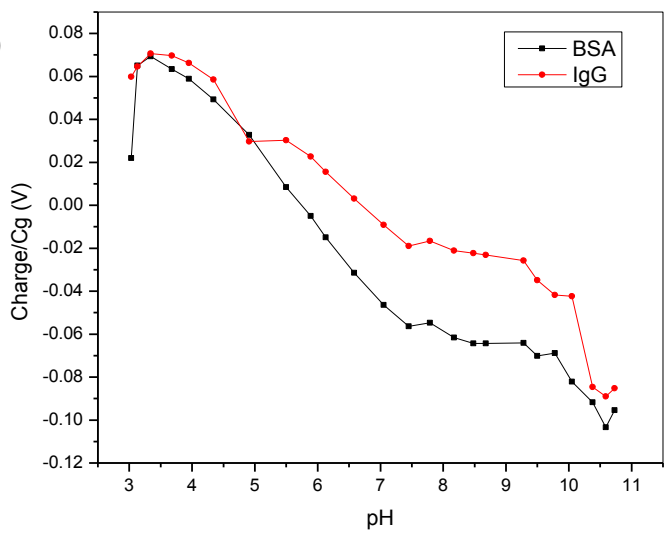

Figure 4. a) Titration curves converted to surface charge. b) Titration curves after elimination of $-\mathrm{OH}$ effect. c) Protein intrinsic titration curve extracted.

\section{Conversion of Surface Potential to Surface Charge}

In order to get the information of surface charges, the zerocharge potential (the surface potential at $\mathrm{pH}$ equal to $\mathrm{pI}$ ) need to be determined first. Although a titration curve does not directly provide its $\mathrm{pI}$, it does give the information of the $\mathrm{pKa}$, and $\mathrm{pKa}, 2$ values where the curve suddenly declines [12]. Notice that the $\mathrm{pKa}_{1}$ and $\mathrm{pKa}_{2}$ values discussed here, represent the apparent behavior of the multi-molecule functionalized surface, rather than being the intrinsic $\mathrm{pKa}, 1$ or $\mathrm{pKa}_{2}$ value of a specific functional group. The $\mathrm{pKa}_{1}$ and 
$\mathrm{pKa}, 2$ values of the three curves in Fig. $3 \mathrm{a}$ and the black curve in Fig. $3 \mathrm{~b}$ were extracted and listed in TALBE II. The red curve in Fig. $3 \mathrm{~b}$ has same $\mathrm{pH}$-dependent behavior of $\mathrm{SiO}_{2}$ surface as the black curve does; therefore further analysis is not necessary. The $\mathrm{pI}$ of each curve can then be calculated according to the following equation:

$$
p I=\frac{p K a,_{1}+p K a,_{2}}{2}
$$

The calculated pIs are also listed in TABLE II. The reference potential of each curve is set at zero-charge potential where the $\mathrm{pH}$ value equals $\mathrm{pI}$. Then the relative surface potential to the zero-charge potential is now representing the surface charge, annotated as 'Charge/Cg (gate capacitance)'. The surface potential of the black curve in Fig. $3 \mathrm{~b}$ and the three curves in Fig. 3a are converted to charge $/ \mathrm{Cg}$, and are entitled the legends of $-\mathrm{OH}$, TESU, TESU+BSA and TESU+IgG, respectively, as shown in Fig. 4 a.

\section{Extraction of Protein Intrinsic Titration Curve}

The net charges on protein are normally more concerned, so a method to extract intrinsic titration curves of proteins is proposed here. Considering that the $-\mathrm{OH}$ groups influence the charges of the curve in Fig. $4 \mathrm{a}$, it is possible to eliminate the effect of $-\mathrm{OH}$ by subtracting the black curve from the others. The charges of the $-\mathrm{OH}$ group are weighted by a factor of 0.4 before subtraction and the result is shown in Fig. 4b.

In Fig. 4b, the black curve represents the charging behavior of the - CHO group only. Since TESU surface is not fully covered by BSA or $\operatorname{IgG}$, the $-\mathrm{CHO}$ charges indicated in the black curve are partially contributing to the titration curves of the BSA (red) and IgG (blue) modified surfaces. The coverage of BSA and IgG are estimated to be around 0.7 , thus, the intrinsic titration curve of each protein is similarly extracted. The black curve is scaled by 0.3 and subtracted from the red and the blue curves, respectively, to obtain the titration curves of pure BSA and IgG. The outcomes are shown in Fig. 4c.

The reliability of the result was verified by the consistency with prior works. The pIs of BSA and mouse IgG obtained from Fig. 4c are 5.7 and 6.6, respectively, which are close to the values appeared in literatures[13]. In addition, both the titration curves of BSA and $\mathrm{IgG}$ show a relatively flat region from $\mathrm{pH} 7.5$ - 9.5, which is commonly observed in typical protein simulation results $[4,12]$. Similar experimental results are also obtained by B. E. Feller[5].

Just for the purpose of protein identification through NGFETs, it is not necessary to obtain the intrinsic protein titration curve with high degree of accuracy. The most significant contribution of this work is the ability to distinguish different proteins by only measuring their apparent titration curves. A standard titration curve can be measured by NGFETs in practical use.

\section{CONCLUSION}

This paper describes a new methodology of measuring protein titration curves by NGFETs. This is a more convenient and reliable method to experimentally obtain titration curves than the conventional ones. In the study two proteins -BSA and mouse IgG - were tested with the apparent titration curves graphed to show the ability of identification. The intrinsic titration curves of the two proteins were also extracted based on proper assumptions and estimations. The resulted curves provide information consistent to both the simulation and experimental results presented in previous researches. In practical use, this method obtains an electronic symbol of a specific protein and shows great potential in the application of protein identification.

\section{ACKNOWLEDGMENT}

This work is partially sponsored by the National Science Foundation (ECCS-0955027, CBET \#1064574, IIP \# 1127761), Texas Instruments Inc., and Texas Medical Consortium. The authors would like to thank Serena Greene for buffer solution preparation.

\section{REFERENCES}

[1] D. Bashford and M. Karplus, "PKAS OF IONIZABLE GROUPS IN PROTEINS - ATOMIC DETAIL FROM A CONTINUUM ELECTROSTATIC MODEL," Biochemistry, vol. 29, pp. 10219-10225, Nov 1990.

[2] M. R. Gunner and E. Alexov, "A pragmatic approach to structure based calculation of coupled proton and electron transfer in proteins," Biochimica Et Biophysica Acta-Bioenergetics, vol. 1458, pp. 63-87, May 2000.

[3] M. J. Ondrechen, J. G. Clifton, and D. Ringe, "THEMATICS: A simple computational predictor of enzyme function from structure," Proceedings of the National Academy of Sciences, vol. 98, pp. 12473-12478, October 23, 20012001.

[4] A. Onufriev, D. A. Case, and G. M. Ullmann, "A Novel View of pH Titration in Biomolecules $\dagger$," Biochemistry, vol. 40, pp. 3413-3419, 2001/03/01 2001.

[5] B. E. Feller, J. T. Kellis, L. G. Cascão-Pereira, C. R. Robertson, and C. W. Frank, "Interfacial Biocatalysis on Charged and Immobilized Substrates: The Roles of Enzyme and Substrate Surface Charge," Langmuir, vol. 27, pp. 250-263, 2011/01/04 2010.

[6]L. Bousse, S. Mostarshed, B. Van Der Shoot, N. F. de Rooij, P. Gimmel, and W. Göpel, "Zeta potential measurements of Ta2O5 and $\mathrm{SiO} 2$ thin films," Journal of Colloid and Interface Science, vol. 147, pp. 22-32, 11// 1991.

[7] R. Kun, M. Szekeres, and I. Dékány, "Isothermal titration calorimetric studies of the $\mathrm{pH}$ induced conformational changes of bovine serum albumin," Journal of Thermal Analysis and Calorimetry, vol. 96, pp. 1009-1017, 2009/06/01 2009.

[8] M. J. Schoning and A. Poghossian, "Recent advances in biologically sensitive field-effect transistors (BioFETs)," Analyst, vol. 127, pp. 1137-1151, 2002.

[9] S. Regonda, R. Tian, J. Gao, S. Greene, J. Ding, and W. Hu, "Silicon multi-nanochannel FETs to improve device uniformity/stability and femtomolar detection of insulin in serum," Biosensors and Bioelectronics, vol. 45, pp. 245-251, 7/15/ 2013.

[10] Z. Pengyuan, L. Yuchen, L. Spurgin, and W. Hu, "pH sensing comparison of vapor and solution APTES coated Si nanograting FETs," in Nanotechnology (IEEE-NANO), 2013 13th IEEE Conference on, 2013, pp. 301-304.

[11] Y. Xinrong, W. R. Frensley, Z. Dian, and H. Wenchuang, "Performance Analysis of Si Nanowire Biosensor by Numerical Modeling for Charge Sensing," Nanotechnology, IEEE Transactions on, vol. 11, pp. 501-512, 2012.

[12] J. J. Ko, L. F. Murga, P. Andre, H. Y. Yang, M. J. Ondrechen, R. J. Williams, et al., "Statistical criteria for the identification of protein active 
sites using theoretical microscopic titration curves," Proteins-Structure Function and Bioinformatics, vol. 59, pp. 183-195, May 2005.

[13] J. F. Foster and L. J. Kaplan, "Isoelectric focussing behavior of bovine plasma albumin, mercaptalbumin, and $\beta$-lactoglobulins $\mathrm{A}$ and $\mathrm{B}, "$ Biochemistry, vol. 10, pp. 630-636, 1971/02/01 1971. 